\section{MYXOID LIPOSARCOMA OF MALE BREAST - AN UNUSUAL PRESENTATION}

Kazi Manzur Kzader¹, A.F.M. Anwar Hossain², Syed Md Akram Hussain ${ }^{3}$, Md. Yakub $\mathrm{Ali}^{4}$

\section{Abstract:}

Myxoid Liposarcoma is the second most common type of Liposarcoma, representing $30-40 \%$ of all Liposarcoma in the extremities, particularly the thighs. The more frequent sites of Myxoid Liposarcoma in decreasing order of frequency are the buttocks, retroperitoneum, trunk, ankles, proximal Limb girdle, head and neck, wrist $(1,2)$. Myxoid Liposarcoma of male breast is an unusual and rare case of breast malignancy.

\section{Summary:}

A 55 years male presented with a mass in the rt. sided Breast. Physical examination shows nothing abnormal except mild anemic with general weakness. There was no palpable regional Lymph node. Finally simple mastectomy was done and histopathological examination revealed Myxoaid Liposarcoma, which is a rare breast tumor.

\section{Case Report:}

A 55 years male presented with firm, irregular and mobile mass in his right breast for few months, which became progressively larger earlier. FNAC from the Lump was done and report was consistent with benign phylloid tumor of breast. Simple mastectomy was done on 08.08.08 and excised mass was sent for biopsy. The biopsy report reveals myxoid Liposarcoma and the patient were referred to National Institute of Cancer Research and Hospital for management. On examination there

1. Associate Professor, Radiation Oncology, National Institute of Cancer Research, Mohakhali, Dhaka.

2. Assistant Professor Surgical Oncology, National Institute of Cancer Research, Mohakhali, Dhaka.

4. Professor and Chairman, Department of Oncology, Bangabandhu Sheikh Mujib Medical University, Dhaka.

3. Assistant Professor, Radiation Oncology, National Institute of Cancer Research, Mohakhali, Dhaka. was no regional Lymphadenopathy, Liver and spleen was not palpable.

He was advised for Laboratory test (CBC, LFT, KFT), X-ray chest P/A view, USG of whole abdomen, ECG. Everything was alright. He was non diabetic and normotensive. The plan of treatment was Chemotherapy (VACA regimen) 6 cycles followed by Local Radiotherapy. The patient has received chemotherapy and Radiotherapy accordingly with supportive measures.

\section{Follow up:}

The patient was advised for regular follow up within 2-3 months interval for $1^{\text {st }}$ year with the investigations like CBC, LFT, KFT, $\mathrm{X}$-ray chest, Ultrasonography of whole abdomen.

\section{Conclusion:}

Various treatment modalities have been advocated, in which surgery is the primary options because it is the first step to remove and confirm the diagnosis. It should be followed by administration of proper chemotherapeutic regimen and radiation treatment depending upon stage of the disease, performance status and also histopathological report.

\section{References:}

1. Sung MS, Kang HS, Sul JS et. al. Myxoid Liposarcoma appearance at MR imaging and histology correlation.

2. Kransdorf MT- Malegnant Softtusive tumor in a large referral population distribution of diagnosis by age sex and location AJR 1995.

3. Jane Dobbs, practical Radiotherapy planning $3^{\text {rd }}$ edition

4. Radiation Oncology, Management Decision- K.S Clitfold CAO, Carlos A Perez, Luther W. Brady.

5. Walter and Millers- Text book of Radiotherapy -Sixth edition.

6. Text book of Radiation Oncology -Principal and practice, Goura K. Rath, Bidtha K. Mohanti.

7. Cancer Management-A Multidisciplinary ApproachRichard Parder- $6^{\text {th }}$ edition. 